\title{
The relationship between abnormal cortical activity in the anterior cingulate gyrus and cognitive dysfunction in patients with end- stage renal disease: a fMRI study on the amplitude of low- frequency fluctuations
}

\author{
Zhengzhang Gu ${ }^{1}$, Haitao Lu ${ }^{1}$, Hua Zhou ${ }^{2}$, Jinggang Zhang ${ }^{1}$, Wei Xing ${ }^{1}$ \\ ${ }^{1}$ Department of Radiology, The Third Affiliated Hospital of Soochow University, Changzhou, China; ${ }^{2}$ Department of Nephrology, The Third \\ Affiliated Hospital of Soochow University, Changzhou, China \\ Contributions: (I) Conception and design: Z Gu, W Xing; (II) Administrative support: W Xing; (III) Provision of study materials or patients: H Zhou; \\ (IV) Collection and assembly of data: J Zhang; (V) Data analysis and interpretation: Z Gu; (VI) Manuscript writing: All authors; (VII) Final approval \\ of manuscript: All authors. \\ Correspondence to: Wei Xing. Department of Radiology, The Third Affiliated Hospital of Soochow University, Changzhou 213000, China. \\ Email: yingxiangfsk@163.com.
}

Background: End-stage renal disease (ESRD) patients often have cognitive dysfunction. The abnormal
changes in the brains of ESRD patients are difficult to detect with routine imaging examination. Cognitive
performance scales are also not an ideal tool because assessments using these scales can be subjective and
might be difficult to administer in some ESRD patients. Functional magnetic resonance has the advantages
of non-radiation, multidirectional imaging, good repeatability. Using resting-state functional magnetic
resonance imaging (rs-fMRI) and an amplitude of low-frequency fluctuation (ALFF) algorithm, this
study characterized spontaneous brain activity and explored its relationship with cognitive function in
ESRD patients. The aim of this study was to provide objective functional imaging evidence to reveal the
pathophysiological mechanism of cognitive dysfunction in ESRD patients. Methods: This study recruited 35 ESRD patients and 25 healthy controls (HC) who were matched to the ESRD group by age, sex, and years of education. All study subjects were examined by Montreal Cognitive Assessment (MoCA) and rs-fMRI. Data Processing \& Analysis of Brain Imaging (DPABI) V4.3 software was used to preprocess the data to obtain ALFF maps. The ALFF value of the cingulate gyrus was compared between the ESRD and HC groups. Subsequently, the correlation between the ALFF value of the cingulate gyrus and the MoCA score was analyzed in ESRD patients.

Results: Compared with the HC group, the ESRD group had a significantly lower MoCA score $(\mathrm{P}<0.05)$. The ALFF values of the anterior and middle cingulate gyri were significantly lower in the ESRD patients (Gaussian random field (GRF) corrected, voxel-level significance: $\mathrm{P}<0.001$, cluster-level significance: $\mathrm{P}<0.05)$. No increased ALFF was observed in any brain regions. The ALFF values of the bilateral anterior cingulate gyri were positively correlated with the MoCA scores ( $r=0.768,0.625$, GRF corrected, voxel-level significance: $\mathrm{P}<0.01$, cluster-level significance: $\mathrm{P}<0.05$ ).

Conclusions: Patients with ESRD showed impaired spontaneous brain activity in the bilateral anterior and middle cingulate gyri, suggesting that ALFF of the anterior cingulate gyrus may be an imaging indicator of cognitive dysfunction in ESRD patients.

Keywords: End-stage renal disease (ESRD); cognitive function; cingulate gyrus; magnetic resonance imaging; amplitude of low frequency fluctuations

Submitted Sep 27, 2020. Accepted for publication Nov 20, 2020.

doi: 10.21037/apm-20-2138

View this article at: http://dx.doi.org/10.21037/apm-20-2138 


\section{Introduction}

End-stage renal disease (ESRD) is the last stage of chronic kidney disease. In recent years, research has found that ESRD can cause functional abnormalities of the central nervous system, especially cognitive dysfunction (1), which may affect the diagnosis and treatment of $\operatorname{ESRD}(2,3)$. The cingulate gyrus is a key brain structure that regulates emotional information, learning, and memory (4). Using functional magnetic resonance imaging (fMRI), a few studies have revealed that the abnormal function of the cingulate gyrus may play a significant role in cognitive dysfunction in ESRD patients $(5,6)$. The amplitude of low-frequency fluctuations (ALFF) in fMRI reflects the energy of signal fluctuations in the low-frequency range $(0.01-0.1 \mathrm{~Hz})$ at the voxel level in blood oxygen level-dependent functional magnetic resonance imaging (BOLD-fMRI) (7). Therefore, ALFF can be used to evaluate regional spontaneous neuronal activity in the brain (8). In this study, we attempted to characterize the neural activity in the cingulate gyrus that is related to cognitive dysfunction in ESRD patients by using an ALFF algorithm, with the hope of providing objective functional imaging evidence for exploring the pathophysiological mechanism underlying ESRD-associated cognitive dysfunction. We present the following article in accordance with the MDAR checklist (available at http://dx.doi.org/10.21037/apm-20-2138).

\section{Methods}

\section{General information}

\section{The ESRD group}

This study included 41 ESRD patients who were treated in our hospital from April 2017 to June 2019. The inclusion criteria were as follows: (I) meting the diagnostic criteria of the American Society of Nephrology for ESRD (9); (II) age between 18 and 60 years old; (III) having undergone dialysis for more than six months; and (IV) right-handedness. The exclusion criteria were (I) a history of neurological or psychiatric diseases, including brain tumors, brain trauma, stroke, schizophrenia, and epilepsy; (II) a history of drug or alcohol dependence; (III) a history of diabetes or cirrhosis; (IV) head motion artifacts interfering experimental data acquisition or measurement; and (V) contraindications for MRI. According to the above exclusion criteria, six ESRD patients were excluded, including one patient with a history of cerebral hemorrhage, one patient with a history of brain trauma, one patient with diabetes, and three patients with motion artifacts that affected experimental data measurement. Finally, the ESRD group comprised 35 patients, including 15 males and 20 females aged 35 to 58 years old, with an average age of $47.60 \pm 6.05$ years.

\section{The healthy control (HC) group}

During the same period, 26 healthy individuals who matched the ESRD patients by age, sex, and years of education were recruited as controls. The inclusion criteria were as follows: (I) age between 18 and 60 years old; (II) physical examination report within the recent six months and no obvious abnormalities in laboratory indicators, electrocardiogram, and B-ultrasound examination; and (III) right-handedness. The exclusion criteria for the $\mathrm{HC}$ group were the same as those for the ESRD group. One case was excluded due to the excessive interference of motion artifacts with data measurement. Finally, the HC group consisted of 25 individuals, including 13 males and 12 females aged 38 to 55 years, with an average age of $47.68 \pm 5.15$ years.

There were no statistically significant differences in sex, age, or years of education between the two groups $(\mathrm{P}>0.05)$. This study was approved by the Ethics Committee of the Third Affiliated Hospital of Soochow University (research number 2017010), and all subjects signed the informed consent form. All procedures performed in this study involving human participants were in accordance with the Declaration of Helsinki (as revised in 2013).

\section{Examinations}

\section{Neuropsychological tests}

The Montreal Cognitive Assessment (MoCA) was used to evaluate the cognitive function of all subjects within one hour before MRI scanning was performed.

\section{MRI}

All MRI examinations were performed using a Philips Achieva 3.0T MRI system equipped with a 16-channel phased-array head coil. Conventional cross-sectional T1-, T2-weighted imaging and coronal T2-weighted fluidattenuated inversion recovery (FLAIR) imaging were performed first to rule out brain structural lesions. Highresolution brain structure images were obtained using a magnetization-prepared rapid gradient echo (MPRAGE) sequence (repetition time $8.0 \mathrm{~ms}$, echo time $3.9 \mathrm{~ms}$, field of view $250 \mathrm{~mm} \times 250 \mathrm{~mm}$, matrix $256 \times 256$, slice thickness $1.0 \mathrm{~mm}$, no gap). Resting-state fMRI (rs-fMRI) was 
performed with a gradient-echo echo-planar imaging (GRE-EPI) sequence (repetition time 2,000 ms, echo time $30 \mathrm{~ms}$, slice thickness $4.0 \mathrm{~mm}$, slice spacing $0.4 \mathrm{~mm}$, field of view $240 \mathrm{~mm} \times 240 \mathrm{~mm}$, matrix $120 \times 117$, flip angle $90^{\circ}$, 250 time points, and 30 slices). During MRI scanning, the patients were asked to remain quiet and close their eyes but stay awake.

\section{Image processing and analysis}

\section{rs-fMRI image preprocessing}

The rs-fMRI image data were preprocessed by using the DPABI V4.3 (http://rfmri.org/dpabi) software package on the MATLAB 2013b platform. The main steps were as follows: (I) format conversion: the DICOM images were converted to the NIFIT format. (II) The data at the first 10 time points were discarded to eliminate interference associated with patients' adaptation to the environment and machine instability. (III) The image data were corrected for slice timing. (IV) The image data were corrected for head motion: Data with a head motion translation greater than $2 \mathrm{~mm}$ or rotation greater than $2^{\circ}$ were discarded. (V) Spatial normalization: The original data were registered with the Montreal Neurological Institute (MNI) template, and the resampling voxel size was set as $3 \mathrm{~mm} \times 3 \mathrm{~mm} \times 3 \mathrm{~mm}$. (VI) The data were treated with linear drift removal and bandpass filtering (frequency $0.01-0.1 \mathrm{~Hz}$ ). (VII) The covariate factors were removed. (VIII) Spatial smoothing: Gaussian smoothing was performed with a full-width at halfmaximum (FWHM) value of $4 \mathrm{~mm}$ to reduce spatial noise.

\section{Calculation of ALFF}

The fast Fourier transform (FFT) algorithm was used to transform the preprocessed time series into the frequency domain to obtain a power spectrum. The ALFF value was obtained by calculating the square root of the power spectrum.

\section{Statistical analysis}

General clinical data were statistically analyzed using SPSS 16.0 software. Age, years of education, and MoCA scores were expressed as $\bar{x} \pm s$, and the differences between the ESRD and HC groups were tested with two-sample $t$ tests. The difference in the sex ratio between the two groups was tested with $\chi^{2}$ tests, and $\mathrm{P}<0.05$ indicated that the difference was statistically significant. Using the DPABI software, the standardized ALFF data were compared with two independent samples $t$ tests and corrected by Gaussian random field (GRF), with $\mathrm{P}<0.001$ at the voxel level and $\mathrm{P}<0.05$ at the cluster level indicating a significant difference. The ALFF values of the cingulate gyrus and the MoCA scores of the ESRD patients were analyzed with Pearson correlation analysis and were GRF corrected, with $\mathrm{P}<0.01$ at the voxel level and $\mathrm{P}<0.05$ at the cluster level indicating a significant difference. Age, sex, and years of education were regarded as covariate factors, and their influence was removed.

\section{Results}

Comparison of general information and cognitive function between the two groups

There were no statistically significant differences in sex, age, and years of education between the two groups $(\mathrm{P}>0.05)$. The MoCA score of the ESRD group was lower than that of the HC group, and the difference between the two groups was statistically significant $(\mathrm{P}<0.05)($ Table 1$)$.

\section{Comparison of the ALFF map of the cingulate gyrus between the ESRD and HC groups}

Compared with the HC group, the ALFF value of the bilateral anterior and middle cingulate gyri was significantly lower in the ESRD group (GRF corrected, voxel-level significance $\mathrm{P}<0.001$ and cluster-level significance $\mathrm{P}<0.05$ ). The ALFF value of the posterior cingulate gyrus did not differ significantly between the two groups $(\mathrm{P}>0.05)$. No detectable increase in the ALFF value was observed in any brain region in the ESRD group (Figure 1 and Table 2).

\section{Correlation analysis between the ALFF value of the cingulate gyrus and cognitive function in ESRD patients}

The ALFF values of the bilateral anterior cingulate gyri were positively correlated with the MoCA score $(r=0.768$, 0.625 , GRF corrected, voxel-level significance $\mathrm{P}<0.01$, cluster-level significance $\mathrm{P}<0.05$ ) (Figure 2, Table 3).

\section{Discussion}

In rs-fMRI studies, ALFF reflects the intensity of neuronal activity in the brain by directly displaying the magnitude change in brain BOLD signals relative to the baseline, and it has been widely used to measure spontaneous functional abnormalities in the brain areas associated with mental 
Table 1 General information and cognitive function of the ESRD and HC groups

\begin{tabular}{lcccc}
\hline Item & ESRD group & HC group & t/ $\chi^{2}$ value & P value \\
\hline Sex (male/female) & $15 / 20$ & $13 / 12$ & 0.490 & 0.484 \\
Age (years) & $47.60 \pm 6.05$ & $47.68 \pm 5.15$ & -0.054 & 0.957 \\
Years of education (years) & $9.97 \pm 2.65$ & $11.16 \pm 2.98$ & -1.625 & 0.110 \\
MoCA (score) & $24.06 \pm 3.86$ & $26.96 \pm 1.34$ & -3.604 & 0.001 \\
\hline
\end{tabular}

ESRD, end-stage renal disease; HC, healthy controls; MoCA, Montreal Cognitive Assessment.

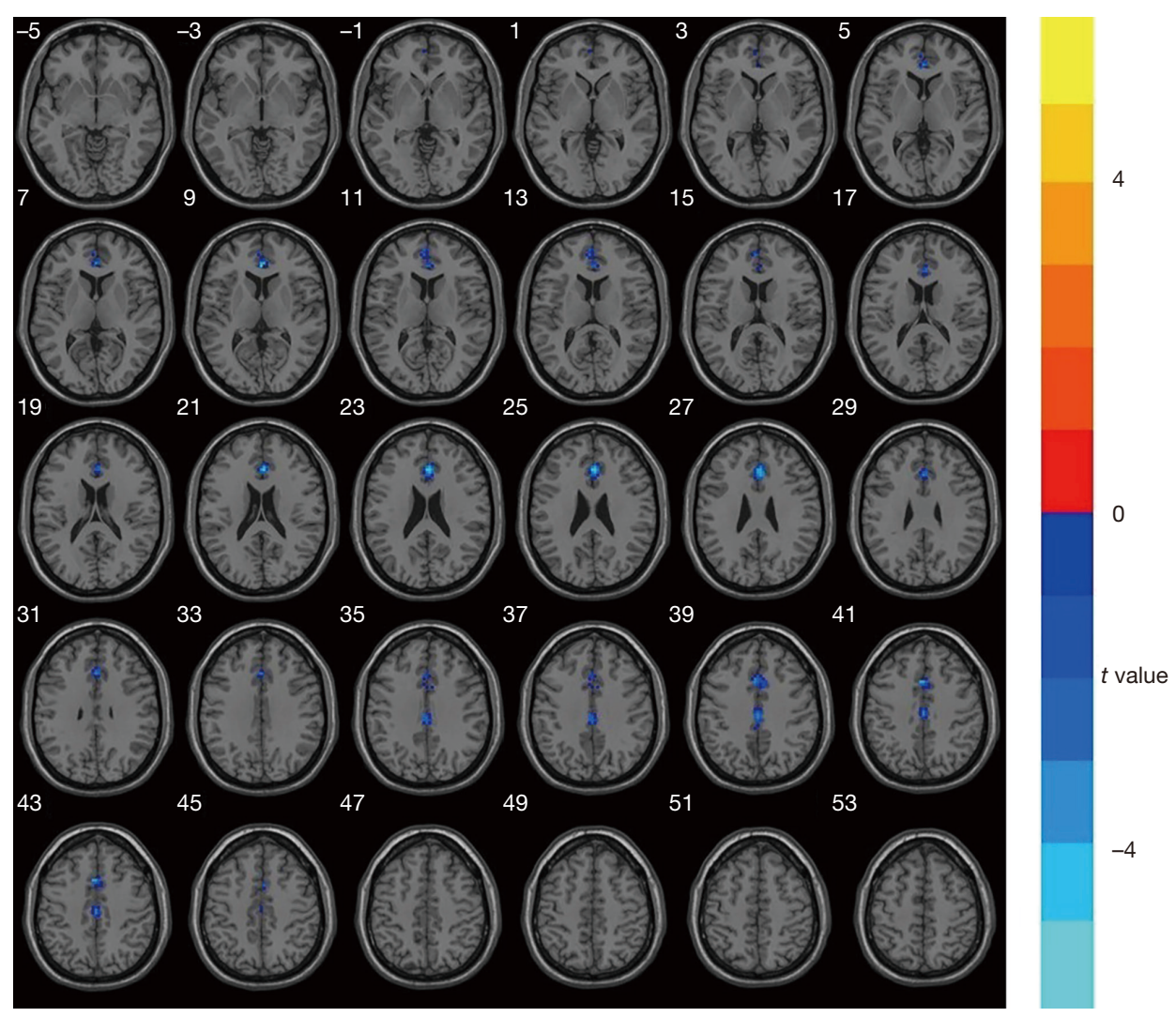

Figure 1 Regions of the anterior and middle cingulate gyri (blue areas) showing decreased ALFF in ESRD patients compared with the HCs. ESRD, end-stage renal disease; HC, healthy controls; ALFF, amplitude of low-frequency fluctuations.

Table 2 Areas in the cingulate gyrus with a decreased mean ALFF in the ESRD group compared with the HC group

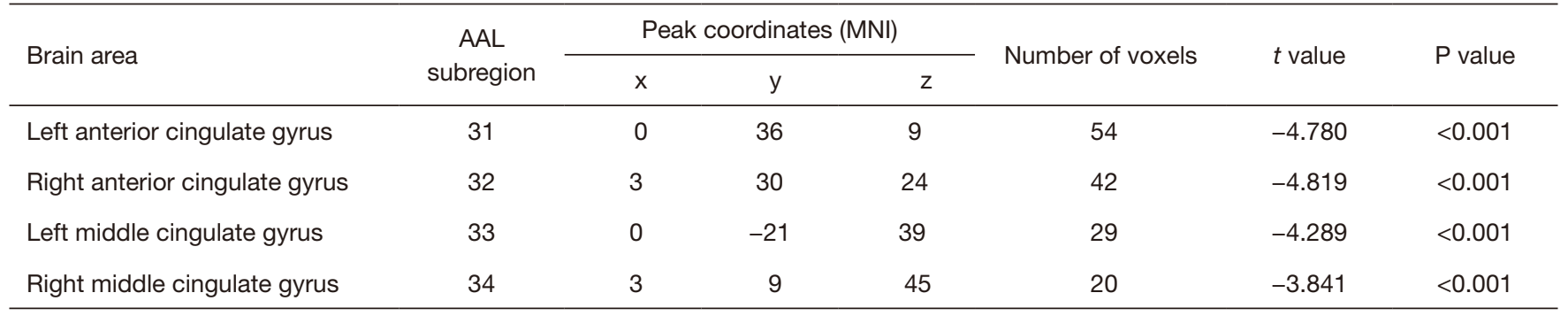

ESRD, end-stage renal disease; HC, healthy controls; ALFF, amplitude of low-frequency fluctuations; AAL, anatomical automatic labeling; MNI, Montreal Neurological Institute. 


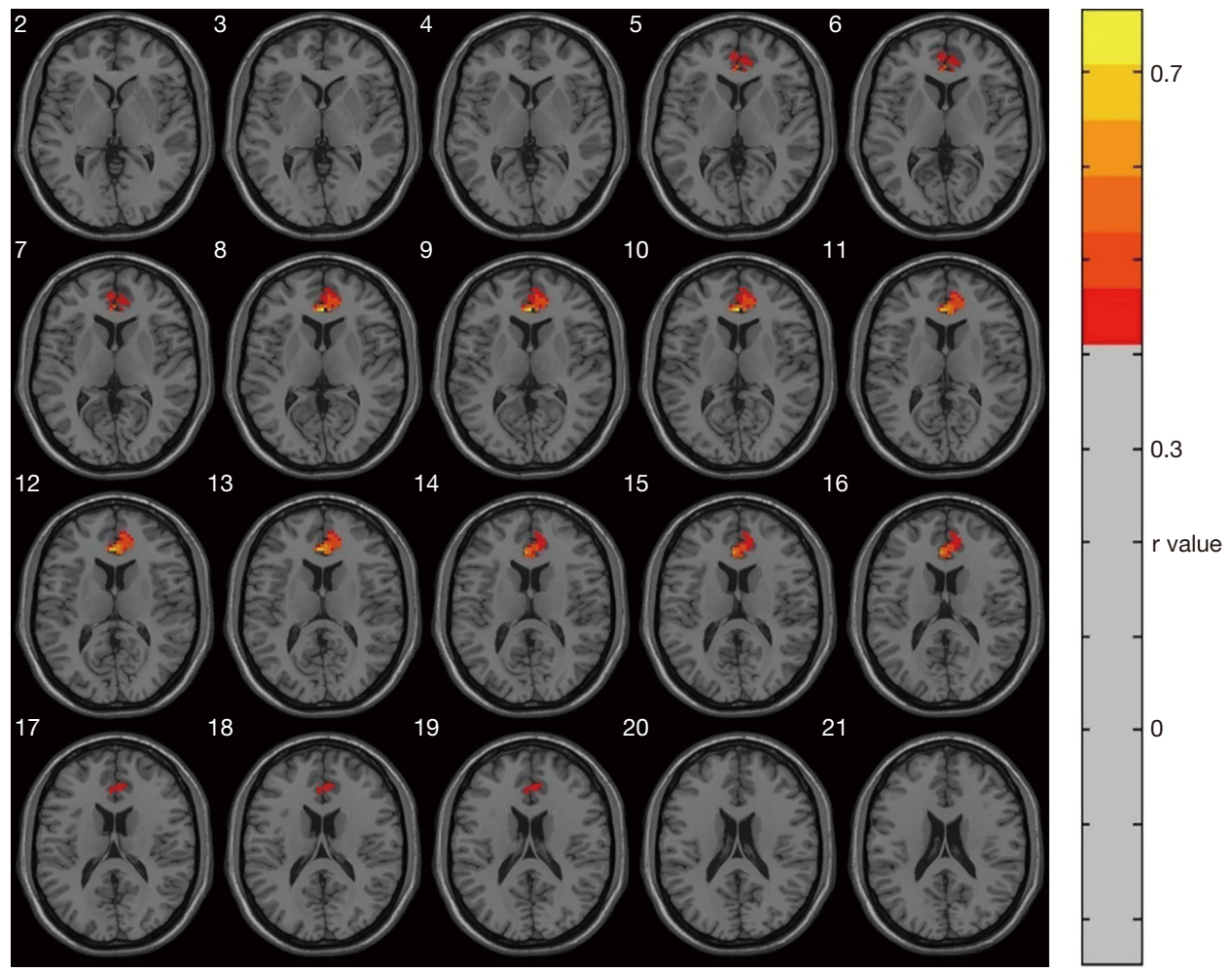

Figure 2 Regions of the anterior cingulate gyrus (red and yellow regions) with ALFF positively correlated with the MoCA score in the ESRD group. ALFF, amplitude of low-frequency fluctuations; MoCA, Montreal Cognitive Assessment; ESRD, end-stage renal disease;

Table 3 Correlation analysis of the ALFF of the cingulate gyrus and the MoCA score in the ESRD group

\begin{tabular}{|c|c|c|c|c|c|c|c|}
\hline \multirow{2}{*}{ Brain area } & \multirow{2}{*}{ AAL subregion } & \multicolumn{3}{|c|}{ Peak coordinates (MNI) } & \multirow{2}{*}{ Number of voxels } & \multirow{2}{*}{ r value } & \multirow{2}{*}{$P$ value } \\
\hline & & $x$ & $y$ & z & & & \\
\hline Left anterior cingulate gyrus & 31 & 0 & 36 & 9 & 24 & 0.768 & $<0.01$ \\
\hline Right anterior cingulate gyrus & 32 & 3 & 36 & 12 & 74 & 0.625 & $<0.01$ \\
\hline
\end{tabular}

ESRD, end-stage renal disease; ALFF, amplitude of low-frequency fluctuations; MoCA, Montreal Cognitive Assessment; AAL, anatomical automatic labeling; MNI, Montreal Neurological Institute.

illness $(10,11)$. Using the ALFF algorithm of rs-fMRI, this study revealed that compared with the HC group, the ESRD group had significantly lower ALFF values in the bilateral anterior and middle cingulate gyri. This indicates that ESRD patients had decreased spontaneous neural activity in the anterior and middle cingulate gyri, suggesting that these brain areas experienced functional damage of the neurons.

In our previous study, independent component analysis of rs-fMRI revealed that ESRD patients exhibited abnormalities in the default-mode network of the brain and had decreased functional connectivity of the posterior cingulate gyrus (12). The finding from the present study that the ALFF values of the bilateral anterior and middle cingulate gyrus were significantly decreased in ESRD patients further supports previous findings. The anterior cingulate gyrus is one of the important structures responsible for the executive function of the brain, and it mainly monitors ongoing directional behaviors and coordinates cognitive processes (13). The present study found that the ALFF of the anterior cingulate gyrus was decreased in the ESRD group, suggesting that ESRD 
patients had weaker spontaneous brain activity in the anterior cingulate gyrus, which is consistent with the findings of $\mathrm{Li}$ et al. (14). In addition, correlation analysis showed that the decrease in ALFF values in the anterior cingulate gyrus was significantly positively correlated with the MoCA score, indicating that the impaired spontaneous activity of neurons in the anterior cingulate gyrus resulted in a gradual decline of cognitive function. The results suggest that the ALFF of the anterior cingulate gyrus may be an objective indicator that can be used to monitor the severity of cognitive dysfunction in ESRD patients. However, there was no significant correlation between the ALFF of the middle cingulate gyrus and cognitive function scores, a finding that may be attributable to the relatively small sample size or the cognitive function scale used. The middle cingulate gyrus is associated with episodic memory and executive function $(15,16)$, and there are close connections between different parts of the cingulate gyrus and between the cingulate gyrus and other brain regions $(17,18)$. Therefore, we speculate that the decreased ALFF value of the middle cingulate gyrus may contribute to cognitive dysfunction in ESRD.

In this study, the ALFF of the posterior cingulate gyrus did not show a significant decrease in ESRD patients, which may be associated with two factors. The first is the severity of disease. One possibility is that damage to the posterior cingulate gyrus occurs later than damage to the anterior and middle cingulate gyri in ESRD patients. Another possibility is that the neural functional impairment in the posterior cingulate gyrus is a manifestation of other factors resulting from the decline of nonspontaneous neural activity in ESRD patients, such as the decrease in functional connectivity found in previous study (12). The second factor is the interference of other diseases. Patients with diabetic nephropathy were excluded from this study. Previous studies have confirmed that the posterior cingulate gyrus is an important structure in the central nervous system that can be impaired in diabetes mellitus $(19,20)$. Therefore, it is likely that the functional impairment of the posterior cingulate gyrus in ESRD patients was caused by confounding factors of both ESRD and diabetes in some existing studies. The question of whether there are any changes in the ALFF of the posterior cingulate gyrus in ESRD patients remains to be further clarified.

This study has some limitations. (I) The sample size was small, and the subjects were not followed up, which might lead to statistical errors in some data. (II) The dialysis methods were not classified and evaluated; as a result, the impact of dialysis methods on brain function may be neglected. Therefore, large-sample longitudinal studies are needed to provide more experimental evidence in the future.

In conclusion, using ALFF analysis of fMRI, this study revealed abnormal spontaneous activity in neurons in the cingulate gyrus of ESRD patients. The results suggest that ALFF analysis can provide an objective imaging basis for investigating abnormal spontaneous neural activity in the brain areas of interest in ESRD, which can improve our understanding of the pathological mechanism underlying ESRD-associated cognitive dysfunction. In addition, the new finding that patients with ESRD have abnormal ALFF values in the middle cingulate gyrus is a supplement to previous research findings.

\section{Acknowledgments}

Funding: Youth Program of National Natural Science Foundation of China(81901696). Project of Changzhou Science and Technology Bureau (CJ20180072). Science and Technology Project for the Young Talents of Changzhou Health and Family Planning Commission (QN201811).

\section{Footnote}

Reporting Checklist: The authors have completed the MDAR checklist. Available at http://dx.doi.org/10.21037/apm-202138

Data Sharing Statement: Available at http://dx.doi. org/10.21037/apm-20-2138

Conflicts of Interest: All authors have completed the ICMJE uniform disclosure form (available at http://dx.doi. org/10.21037/apm-20-2138). The authors have no conflicts of interest to declare.

Ethical Statement: The authors are accountable for all aspects of the work in ensuring that questions related to the accuracy or integrity of any part of the work are appropriately investigated and resolved. This study was reviewed and approved by The Third Affiliated Hospital of Soochow University ethical review board (research number 2017010), and all patients signed informed consent before enrollment. All procedures performed in this study involving human participants were in accordance with the Declaration of Helsinki (as revised in 2013). 
Open Access Statement: This is an Open Access article distributed in accordance with the Creative Commons Attribution-NonCommercial-NoDerivs 4.0 International License (CC BY-NC-ND 4.0), which permits the noncommercial replication and distribution of the article with the strict proviso that no changes or edits are made and the original work is properly cited (including links to both the formal publication through the relevant DOI and the license). See: https://creativecommons.org/licenses/by-nc-nd/4.0/.

\section{References}

1. Foster R, Walker S, Brar R, et al. Cognitive Impairment in Advanced Chronic Kidney Disease: The Canadian Frailty Observation and Interventions Trial. Am J Nephrol 2016;44:473-80.

2. Weiner DE, Seliger SL. Cognitive and physical function in chronic kidney disease. Curr Opin Nephrol Hypertens 2014;23:291-7.

3. Kurella Tamura M, Yaffe K. Dementia and cognitive impairment in ESRD: diagnostic and therapeutic strategies. Kidney Int 2011;79:14-22.

4. Peru A, Pavesi G, Campello M. Impairment of executive functions in a patient with a focal lesion in the anterior cingulate cortex. Evidence from neuropsychological assessment. Funct Neurol 2004;19:107-11.

5. Ni L, Wen J, Zhang LJ, et al. Aberrant default-mode functional connectivity in patients with end-stage renal disease: a resting-state functional MR imaging study. Radiology 2014;271:543-52.

6. Zhang XD, Wen JQ, Xu Q, et al. Altered long- and shortrange functional connectivity in the patients with endstage renal disease: a resting-state functional MRI study. Metab Brain Dis 2015;30:1175-86.

7. Zang YF, He Y, Zhu CZ, et al. Altered baseline brain activity in children with ADHD revealed by resting-state functional MRI. Brain Dev 2007;29:83-91.

8. He Z, Deng W, Li M, et al. Aberrant intrinsic brain activity and cognitive deficit in first-episode treatmentnaive patients with schizophrenia. Psychol Med 2013;43:769-80.

9. Saran R, Robinson B, Abbott KC, et al. US Renal Data System 2018 Annual Data Report: Epidemiology of Kidney Disease in the United States. Am J Kidney Dis 2019;73:A7-A8.

10. Hoptman MJ, Zuo XN, Butler PD, et al. Amplitude of low-frequency oscillations in schizophrenia: a resting state fMRI study. Schizophr Res 2010;117:13-20.

11. Zhou G, Liu P, Wang J, et al. Fractional amplitude of lowfrequency fluctuation changes in functional dyspepsia: a resting-state fMRI study. Magn Reson Imaging 2013;31:996-1000.

12. Lu H, Gu Z, Xing W, et al. Alterations of default mode functional connectivity in individuals with end-stage renal disease and mild cognitive impairment. BMC Nephrol 2019;20:246.

13. Elliott R. Executive functions and their disorders. Br Med Bull 2003;65:49-59.

14. Li P, Ding D, Ma XY, et al. Altered intrinsic brain activity and memory performance improvement in patients with end-stage renal disease during a single dialysis session. Brain Imaging Behav 2018;12:1640-9.

15. Lim EC, Tan JJ, Ong BK, et al. Generalised myoclonus evolving into epilepsia partialis continua due to a cingulate gyrus lesion: case report and review of the literature. Parkinsonism Relat Disord 2004;10:447-9.

16. Clement F, Gauthier S, Belleville S. Executive functions in mild cognitive impairment: emergence and breakdown of neural plasticity. Cortex 2013;49:1268-79.

17. Tamburin S, Cacciatori C, Bonato C, et al. Cingulate gyrus tumor presenting as panic attacks. Am J Psychiatry 2008;165:651-2.

18. San Pedro EC, Mountz JM, Ojha B, et al. Anterior cingulate gyrus epilepsy: the role of ictal rCBF SPECT in seizure localization. Epilepsia 2000;41:594-600.

19. Zhang Z, Zhang B, Wang X, et al. Altered Odor-Induced Brain Activity as an Early Manifestation of Cognitive Decline in Patients With Type 2 Diabetes. Diabetes 2018;67:994-1006.

20. Chen YC, Jiao Y, Cui Y, et al. Aberrant brain functional connectivity related to insulin resistance in type 2 diabetes: a resting-state fMRI study. Diabetes Care 2014;37:1689-96.

Cite this article as: $\mathrm{Gu}$ Z, Lu H, Zhou H, Zhang J, Xing W. The relationship between abnormal cortical activity in the anterior cingulate gyrus and cognitive dysfunction in patients with end-stage renal disease: a fMRI study on the amplitude of low-frequency fluctuations. Ann Palliat Med 2020;9(6):4187-4193. doi: 10.21037/apm-20-2138 